Eine pluralistische makroökonomische Analyse

\title{
Rahmenbedingungen einer Postwachstumsökonomie
}

\author{
Eine Wirtschaft jenseits des Wachstums be- \\ darf grundlegender makroökonomischer \\ Veränderungen. Sie betreffen das Steuersystem, \\ die Unternehmensformen, die Verteilung des \\ Wohlstands und die Arbeitszeiten. Doch auch \\ Werbung und Bildung müssen sich wandeln. \\ Von Steffen Lange
}

$E^{s}$ s gibt nur wenige Analysen, die aus makroökonomischer Perspektive untersuchen, wie eine Postwachstumsökonomie (PWÖ) aussehen könnte. Zentrale Merkmale einer PWÖ sind Null- oder negatives Wirtschaftswachstum sowie ein sinkender Ressourcenverbrauch. Darüberhinaus ist sie geprägt von einer geringeren Einkommensungleichheit, mehr demokratischer Mitbestimmung und einer gleichmäßigeren Verteilung von Lohn- und Reproduktionsarbeit zwischen den Geschlechtern [1]. In diesem Artikel wird gezeigt, welche makroökonomischen Rahmenbedingungen aus der Sicht unterschiedlicher makroökonomischer Theorien (neoklassische, postkeynesianische, neomarxistische) für eine PWÖ notwendig sind. Zunächst wird gezeigt, wie sich die Zusammensetzung der Produktionsfaktoren verändern würde. Im zweiten Schritt wird untersucht, welche Rahmenbedingungen zu einer solchen Veränderung führen würden.

\section{Entwicklung der Produktionsfaktoren}

Produktionsfaktoren - also Arbeit, physisches Kapital und natürliche Ressourcen - sind ein guter Ausgangspunkt für die Untersuchung. Wie haben sich diese in der Vergangenheit entwickelt und wie würden sie sich in einer PWÖ entwickeln? Die meisten modernen Produktionsfunktionen beinhalten folgende Faktoren:

$Y=T(K, L, R)$

Die Produktionsmenge (Y) ist abhängig von der Art der eingesetzten Technologie ( $\mathrm{T}$ ) und der Mengen des eingesetzten Kapitals (K), der Arbeit (L) und der natürlichen Ressourcen (R). Die Technologie spielt hierbei eine besondere Rolle: Sie bestimmt die Produktivität und die Zusammensetzung der anderen Faktoren.

Seit der industriellen Revolution ist die Produktion pro Kopf in vielen Ländern stark angestiegen. Grund hierfür ist per De- finition eine Erhöhung der durchschnittlichen Arbeitsproduktivität. Folgende Faktoren spielen dabei eine Rolle: Erstens, ein erhöhter Einsatz von physischem Kapital im Produktionsprozess, zweitens ein erhöhter Einsatz von natürlichen Ressourcen, drittens die Verwendung neuer Technologien und viertens qualifiziertere Arbeitskräfte. Stark verallgemeinert war also die Entwicklung der Wirtschaft seit der industriellen Revolution geprägt von: mehr Maschinen, mehr Ressourcenverbrauch, anderen Technologien und qualifizierteren Arbeitskräften.

Auf der Ebene der Produktionsfaktoren sind zwei Entwicklungen durch die Definition einer nachhaltigen PWÖ vorausgesetzt. Einerseits geht der Ressourcenverbrauch in einer PWÖ zurück und andererseits stagniert oder sinkt die Produktion pro Kopf.

Um diese Ziele zu erreichen, gibt es bezogen auf die Produktionsfunktion sehr unterschiedliche Entwicklungskombinationen der einzelnen Faktoren. Solange der Ressourcenverbrauch zurückgeht, können die anderen Faktoren scheinbar alle zunehmen oder abnehmen oder eine Kombination davon. Ob dies zu einem Rückgang der Produktion führt, kommt auf die Größenverhältnisse der Veränderungen an.

Theoretisch scheint also vieles möglich. Allerdings hängen die Entwicklungen der einzelnen Produktionsfaktoren in der Realität voneinander ab. Beispielsweise ist die Produktion von Kapitalgütern mit Ressourcenverbrauch verknüpft. Eine zentrale Frage ist daher, wie die Entwicklungen der Faktoren miteinander in Verbindung stehen. Hierzu sind in der Literatur mindestens drei zentrale Theorierichtungen $\mathrm{zu}$ unterscheiden.

\section{Produktionsfaktoren und Postwachstum}

Autor(inn)en der Neoklassik gehen prinzipiell davon aus, dass die verschiedenen Faktoren sich unabhängig voneinander entwickeln können [2]. Dies hängt eng mit der Frage der Substituierbarkeit und dem Vertrauen in technologischen Fortschritt zusammen. In neoklassischen Analysen können alle Faktoren, also auch natürliche Ressourcen, durch andere Faktoren ersetzt werden. Die Frage ist, zu welchem Grad dies möglich ist: „If it is very easy to substitute other factors for natural resources, then there is in principle no problem. The world can, in effect, get along without natural resources, so exhaustion is just an event, not a catastrophe. [...] If, on the other hand, real output per unit of resources is effectively bounded - cannot exceed some upper limit of productivity which is in turn not too far from where we are now - then catastro- 
phe is unavoidable“ (Solow 1974). Diese prinzipielle Möglichkeit führt viele Autor(inn)en dieser Richtung zu der Aussage, dass eine Entkopplung des Wachstums vom Ressourcenverbrauch möglich ist.

Für die Untersuchung einer PWÖ von größerer Bedeutung: Aus dieser Sicht sind alle möglichen Kombinationen von $\mathrm{Zu}$ und Abnahme der Determinanten der Produktionsfunktion vorstellbar, um ein Nullwachstum oder eine Schrumpfung mit gleichzeitiger Abnahme der natürlichen Ressourcen zu erreichen.

An dieser Theorie ist erstens zu kritisieren, dass oft sehr optimistische Annahmen bezüglich der Substituierbarkeit der Faktoren gemacht werden. Es wird behauptet, sie seien nicht nur prinzipiell substituierbar, sondern dies könne auch zügig stattfinden (Stern 1997). Zweitens bezieht sie nicht mit ein, dass die Herstellung von physischem Kapital ebenfalls natürliche Ressourcen braucht - und daher natürliche Ressourcen nicht primär durch physisches Kapital ersetzt werden können (Kümmel 2011).

Im Postkeynesianismus werden die Verhältnisse der Produktionsfaktoren meist als verhältnismäßig starr angesehen, Substitution ist nur sehr bedingt möglich. Eine Abnahme des Verbrauchs der natürlichen Ressourcen würde also tendenziell mit einer Abnahme von Kapital und Arbeit einhergehen. Wenn die Technologie gleichzeitig (graduell) arbeits-, ressourcen- und kapitaleffizienter wird, könnte dies ein Nullwachstum mit abnehmendem Ressourcenverbrauch, abnehmender Arbeitsmenge und abnehmendem Kapitaleinsatz bedeuten.

Postkeynesianische Theorien sind meist nicht darauf ausgelegt zu erklären, wie viel von welchem Produktionsfaktor in der Produktion verwendet wird, weil sie sich auf die kurze Frist fokussieren. Für eine mittel- bis langfristige Analyse ist die Annahme der unveränderbaren Verhältnisse der Faktoren nicht zielführend.

In den meisten dieser Arbeiten des Neomarxismus und der Ökologischen Ökonomik wird die historische Zunahme der Arbeitsproduktivität als untrennbar von der Akkumulation von Kapital und der Nutzung von natürlichen Ressourcen betrachtet. Die Arbeitsproduktivität ist abhängig vom Energieverbrauch und von physischem Kapital (Kümmel 2011). Physisches Kapital besteht aus natürlichen Ressourcen, ist also mit deren Verbrauch verbunden. Wenn die Nutzung natürlicher Ressourcen in bedeutendem Ausmaße verringert wird, nimmt daher sowohl der Ressourcenverbrauch als auch das physische Kapital ab. Hierdurch geht die Arbeitsproduktivität zwangsläufig zurück. Eine PWÖ wäre also durch den Einsatz von weniger natürlichen Ressourcen und weniger physischem Kapital pro geleisteter Arbeitsstunde gekennzeichnet.

\section{Synthese}

Die Theorien liefern uns sehr unterschiedliche Erkenntnisse, die nicht unbedingt im Widerspruch stehen müssen. Die Neoklassik zeigt, was theoretisch möglich ist und in wel- che Richtung man versuchen kann, die Wirtschaft zu lenken. Dazu gehört auch die Substitution von Ressourcenverbrauch durch Arbeitseinsatz und ressourceneffizientere Technologien. Theoretisch sind Technologien denkbar, die sehr unterschiedliche Kombinationen von Inputfaktoren und Produktivitäten beinhalten können.

Der Postkeynesianismus liefert uns die Restriktion für die unterschiedlichen vorstellbaren Welten für die kurze und mittlere Frist. Technologien verändern sich in kurzer Zeit nicht grundlegend. Die Konsequenz daraus ist, dass der Ressourcenverbrauch kurzfristig nur stark verringert werden kann, wenn die Produktion abnimmt.

Neomarxistische Theorien und Theorien der ökologischen Ökonomik geben uns Einsicht in die lange Frist. Bisher war die langfristige Zunahme der Arbeitsproduktivität mit einer starken Expansion der Nutzung fossiler Energieträger verbunden. Diese Option steht nicht mehr zur Verfügung. Selbst wenn es theoretisch möglich erscheint, durch technologische Erneuerungen die Arbeitsproduktivität vom Ressourcenverbrauch zu entkoppeln - so etwas hat bisher noch nicht stattgefunden und es ist sehr unsicher, ob es klappen kann. Zukunftsplanung sollte nicht zentral auf einer überaus optimistischen und unsicheren Annahme basieren.

Hieraus ergibt sich ein differenziertes Bild, wie sich Inputfaktoren in einer PWÖ verändern würden: In den Sektoren, in denen dies möglich ist, wird der Verbrauch natürlicher Ressourcen durch Arbeitskraft in der Produktion substituiert. In den Sektoren, in denen eine solche Substitution wegen der bestehenden Produktionsmethoden nur in geringem Maß möglich ist, wird die Produktion verringert. Langfristig müssen Strategien entwickelt werden, wie mit sehr wenigen fossilen Energieträgern und geringerem materiellen Wohlstand umgegangen werden kann.

\section{Makroökonomische Rahmenbedingungen}

Bis hierher wurde skizziert, wie eine PWÖ bezüglich der Produktionsfaktoren aussieht. Die daran anschließende Frage lautet, welche Rahmenbedingungen für eine solche Entwicklung nötig sind.

In einer PWÖ kommen Produktionsmethoden zum Einsatz, die den Produktionsfaktor Arbeit verhältnismäßig mehr und natürliche Ressourcen weniger nutzen. Eine solche Veränderung der Produktion kann durch unterschiedliche Mechanismen hervorgerufen werden. (1) Veränderung der Zusammenstellung der Güter, die produziert und konsumiert werden: Zur Verfügung stehendes Einkommen wird verstärkt für arbeits- statt ressourcenintensive Produkte ausgegeben. (2) Umstellung auf andere bereits existierende Produktionsmethoden: Aus der Auswahl existierender Produktionsmethoden werden arbeits- statt ressourcenintensive Methoden ausgewählt. (3) Entwicklung neuer Produktionsmethoden: In Zukunft werden nicht arbeitssparende, sondern ressourcensparende Produktionsmethoden entwickelt (Jaffe et al. 2003). 


\section{Die Angebotsseite}

Diese Veränderungen können durch eine Vielzahl wirtschaftspolitischer Rahmenbedingungen und veränderter Verhaltensmuster befördert werden. Zentral hierbei ist die Veränderung der relativen Preise von Ressourcenverbrauch und Arbeit. Derzeit werden in Deutschland zwei Drittel der Staatseinnahmen über den Faktor Arbeit und lediglich etwa zehn Prozent über die Besteuerung natürlicher Ressourcen eingenommen (Ludewig et al. 2010). Eine höhere Besteuerung von Ressourcenverbrauch und eine geringere Besteuerung von Lohneinkommen würden Ressourcen relativ zur Arbeit verteuern. Bei diesen Überlegungen muss ebenfalls die Finanzierung der Sozialsysteme mitgedacht werden, da diese derzeit primär über Lohnnebenkosten finanziert werden und damit den Faktor Arbeit verteuern. Diese Maßnahmen würden alle drei zuvor skizzierten Mechanismen beeinflussen: Umweltfreundliche Produkte werden billiger und damit attraktiver $\mathrm{zu}$ konsumieren, arbeitsintensive Produktionsmethoden werden wettbewerbsfähig und Investitionen in die Innovation umweltschonender Produktionsmethoden werden profitabel.

Ein weiteres zentrales Phänomen sind positive Skaleneffekte. Derzeit ist die Produktion großer Mengen in vielen Bereichen pro Stück billiger als die Produktion kleiner Mengen. Dies hat zur Folge, dass Unternehmen ihre Gewinne in neue und größere Produktionsanlagen investieren müssen, da sie sonst vom Markt verdrängt werden (Marx 1867). Wenn alle Unternehmen versuchen, viel zu investieren, um ihre Produktion auszuweiten, führt dies auf makroökonomischer Ebene zu einer Erhöhung der Nachfrage nach Investitionsgütern und damit zu Wachstum (Binswanger 2013).

Die Frage ist nun, wie sich eine Verteuerung von Ressourcen und Kapital gegenüber Arbeit auf diesen Prozess auswirken würde. Die Vergangenheit hat gezeigt, dass ressourcenintensive Produktion billiger ist, wenn man große Mengen produziert. Gilt dasselbe für arbeitsintensive Produktion? Es gibt Gründe, die dagegen sprechen. Als zwei der Hauptgründe für positive Skaleneffekte werden die Verteilung von Fixkosten auf eine größere Anzahl Produkte und die Zunahme von Energieverwendung im Produktionsprozess genannt. Diese beiden Effekte würden durch eine veränderte Besteuerung abnehmen. Andere Effekte würden allerdings bestehen bleiben, sodass offen bleibt, wie sehr sich der Investitionsanreiz verändern würde. Gemeinsam mit der Einführung anderer Unternehmensformen, würde der Anreiz, in Produktionsausweitung zu investieren, aber in jedem Fall abnehmen.

Ein weniger offensichtliches, jedoch wichtiges Instrument ist die öffentliche Förderung von Forschungsprojekten. Die Entwicklung arbeitsintensiver und umweltfreundlicher Produktionsmethoden kann durch Forschung gefördert werden. Ein weiterer Ansatzpunkt ist das Bildungs- und Ausbildungssystem. Innovationen treten verstärkt in den Bereichen auf, in denen die nötigen Produktionsfaktoren, also auch qualifizierte Arbeitskräfte, in ausreichendem Maße zur Verfügung stehen
(Acemoglu 2002). Gibt es viele Arbeitskräfte mit den nötigen Fähigkeiten für arbeitsintensive Produktionsmethoden, wird auch eher in diese investiert.

\section{Kollektive Unternehmensformen}

Technologisierung findet nicht nur aus Kosten-, sondern auch aus Machtgründen statt. Um die Verhandlungsposition von Arbeitnehmer(inne)n zu schwächen, werden tendenziell Produktionsmethoden eingeführt, die geringe Qualifizierung benötigen [3]. Damit können Lohnforderungen von hochqualifizierten, und dadurch schwer austauschbaren Arbeitskräften entgegengewirkt werden (Braverman 1998). Auf der anderen Seite kann man beobachten, dass Unternehmen mit demokratischeren Entscheidungsstrukturen und damit einem Anspruch an geringere Machtgefälle auch andere Produktionsmethoden einsetzen. Unternehmensstrukturen, in denen die Arbeitnehmer(innen) die Entscheidungen treffen, produzieren daher tendenziell arbeitsintensiver und entlassen seltener Arbeitskräfte (Burdín/Dean 2012). Die Förderung solcher Unternehmensformen wäre daher ein wichtiger Bestandteil einer PWÖ.

Ein weiteres Argument, das Unternehmensformen mit Wachstum verbindet, betrifft die Interessen der Kapitaleigner(innen). Gehört das Unternehmen Menschen, die neben ihrem Eigentum keine andere Verbindung zum Unternehmen haben, ist ihr primäres Interesse die Steigerung ihrer Kapitalanlage. Der Wert eines Unternehmensanteils steigt mit der Größe des Unternehmens. Daher haben Aktionäre ein zentrales Interesse daran, dass ihr Unternehmen wächst (Binswanger 2013). Unternehmen versuchen zu wachsen, indem sie in größere Produktion investieren. Auf makroökonomischer Ebene führt dies zu einem Anstieg der Nachfrage und zu Wachstum. In Unternehmensformen, in denen Stakeholder die Entscheidungen treffen, fließen weitere Interessen in die Entwicklung des Unternehmens ein. Hierdurch wird der Fokus auf Unternehmenswachstum geschwächt.

Daneben gibt es weitere Argumente für eine demokratischere Organisation von Unternehmen. Zuvorderst steht hier das Argument, dass in einer demokratischen Gesellschaft auch die Wirtschaft demokratisch organisiert werden sollte, um allen Mitgliedern der Gesellschaft eine möglichst gleichberechtigte Gestaltung ihrer Lebensumstände zu ermöglichen (Krätke 2009).

\section{Die Nachfrageseite}

Neben der Nachfrage nach Investitionsgütern durch die Unternehmen spielt die Nachfrage nach Konsumgütern durch die Konsument(inn)en eine zentrale Rolle für die Höhe und $\mathrm{Zu}$ sammensetzung der Gesamtnachfrage und damit der Produktion. Die zuvor angeführten Umstellungen würden auch auf Nachfrageseite zu Veränderungen führen. Eine Verschiebung der relativen Preise macht ökologisch nachhaltigen Konsum attraktiver und eine Umstellung auf arbeitsintensive Pro- 
duktion verringert die durchschnittliche Arbeitsproduktivität und damit das durchschnittliche reale Einkommen. Folgende Aspekte sind zusätzlich zentral, um die Nachfrageseite insgesamt geringer ausfallen zu lassen und arbeitsintensive statt ressourcenintensive Produktion zu unterstützen.

Umverteilung: Umverteilung kann eine große Rolle für die Verringerung relativen Konsums spielen: Menschen konsumieren zunehmend nicht wegen des Produkts an sich, sondern weil es ein Symbol für Status oder Zugehörigkeit ist. Würde man finanzielle Ungleichheiten verringern, würde die Notwendigkeit für solchen Konsum abnehmen (Rajan 2011). Umverteilung hat allerdings auch gegenteilige Effekte: Beispielsweise haben Menschen mit niedrigen Einkommen eine höhere Konsumquote. Das wichtigste Argument ist jedoch, dass bei einer Verringerung des durchschnittlichen realen Einkommens die Einkommensungleichheit verringert werden muss, wenn eine Zunahme der Anzahl der Menschen mit sehr geringen Einkommen verhindert werden soll.

Arbeit: Wenn vermehrt arbeitsintensiv produziert wird und die Nachfrage nach arbeitsintensiven Gütern wächst, steigt auch die Nachfrage der Unternehmen nach Arbeit. Bei gleichbleibender Produktion würde also die Arbeitsmenge steigen [4]. Wenn die Produktion abnimmt, kommt es auf das Größenverhältnis zwischen Produktionsrückgang und Arbeitsintensivierung der Produktion an, ob die Arbeitsmenge steigt oder fällt.

Die Kausalität verläuft allerdings tendenziell in die gegenteilige Richtung: Wenn Menschen mehr arbeiten, verdienen sie mehr und haben mehr Geld zum konsumieren. Durch vorteilhafte rechtliche Rahmenbedingungen und finanzielle Anreize zu Teilzeitarbeit würden Menschen sich eher gegen viel Lohnarbeit, Einkommen und Konsum und für mehr andere Arbeit und Freizeit entscheiden. Es wird oft argumentiert, dass in einer PWÖ mehr repariert, selbst produziert und nichtkommerziell produziert werden soll (Paech 2013). Teilzeitarbeit würde Menschen die Zeit für diese Tätigkeiten zur Verfügung stellen. Um eine gleichmäßigere Verteilung von Lohn- und Reproduktionsarbeit zwischen den Geschlechtern zu erreichen, müssten vorrangig Männer ihre Arbeitszeit verkürzen, da diese im Durchschnitt weitaus mehr Lohnarbeit nachgehen als Frauen - und weniger Reproduktionsarbeit übernehmen (Biesecker 1999).

Insgesamt gibt es im Bereich Arbeit somit ein Spannungsverhältnis: Eine arbeitsintensivere Produktion erhöht die Nachfrage nach Arbeit, gleichzeitig sind Arbeitszeitverkürzungen notwendig, um suffiziente Lebensstile zu ermöglichen. Umso größer die Substituierung von Ressourcen durch Arbeit und die Zunahme von Teilzeitarbeit ausfallen, desto größer würde der Rückgang der Produktion sein.

Werbung: Durch Werbung werden Menschen dazu angeregt, mehr der bereits bekannten Produkte zu konsumieren, und Wünsche nach neuen Produkten werden geweckt (Galbraith/Crook 1958). Neben Werbung spielt die Darstellung normaler Lebensweisen in den Medien eine gewichtige Rolle für die Konsumentscheidungen. Wenn durch Medien suggeriert wird, dass der materielle Lebensstandard der Oberschicht normal ist, versuchen Menschen dieses Konsumniveau zu erreichen (Schor 1999). Geringere Werbung und eine realistische Darstellung normaler Lebensweisen in den Medien würden zu einem geringeren Konsumlevel beitragen.

Bildung: Bildung hat ebenfalls einen großen Einfluss auf das Konsumverhalten. Um einen wie zuvor skizzierten Konsum zu fördern, würden konsumkritische und auf nachhaltigen Konsum ausgerichtete Perspektiven stärker in den Fokus von Bildung gerückt. Strategien für einen nachhaltigen Lebensstil könnten auch in Bildungskontexten erprobt und eingeübt werden. Bildung spielt darüber hinaus eine zentrale Rolle für die Förderung der Akzeptanz vieler anderer Veränderungen, die in diesem Artikel beschrieben wurden.

\section{Internationale Wettbewerbsfähigkeit}

Gegen viele der Vorschläge, die sich aus obiger Analyse ergeben haben, wird der Einwand erhoben, dies könne man unter der Bedingung internationalen Wettbewerbs nicht durchführen: Vermögende würden ihr Geld ins Ausland verschieben, wenn es besteuert würde; Hochqualifizierte wanderten aus, wenn sie weniger verdienen würden; Unternehmen verlagerten ihre Produktion, wenn ihre Gewinne besteuert würden; die deutsche Wirtschaft könnte ihre Produkte nicht mehr international verkaufen, wenn Ressourcenverbrauch stärker besteuert würde, und so weiter.

Es gibt drei Antworten auf diese Einwände: (1) Spielräume nutzen: Zunächst ist zu fragen, ob diese Argumente stimmen. Pauschal lässt sich darauf keine Antwort geben. Es gibt aber viele Autor(inn)en, die zu dem Ergebnis kommen, dass der Spielraum einzelner Staaten, ihre Wirtschaftspolitik zu bestimmen, größer ist, als oftmals angenommen (Krugman 1994). Dies ist insbesondere der Fall in Deutschland, da hier derzeit hohe Exportüberschüsse erwirtschaftet werden. Aus makroökonomischer Perspektive ist es daher sogar wünschenswert, dass deutsche Unternehmen insgesamt weniger wettbewerbsfähig würden, um internationale Ungleichgewichte zu verringern (Stockhammer 2011). (2) Einzelstaatliche Spielräume erweitern: Wenn einzelne Staaten ihre Handlungsmöglichkeiten zu verlieren drohen, gilt es, sie zu sichern, beziehungsweise zu erweitern. Zusätzliche Liberalisierung von Güter- und Kapitalflüssen ist $\mathrm{zu}$ vermeiden oder einzuschränken (Rodrik 2011). (3) Internationale Regeln: Auf internationaler Ebene können Regeln eingeführt werden, welche die vorgezeichnete Entwicklung unterstützen. Mindeststeuersätze können länderübergreifend beschlossen, Sozial- und Ökostandards eingeführt werden (Stiglitz 2007).

\section{Fazit}

In einer PWÖ würden neue Technologien zum Einsatz kommen, die verhältnismäßig weniger Ressourcen und mehr Arbeit benötigen. Insgesamt würden Produktion und Konsum ge- 
ringer ausfallen, als es derzeit in den Industrieländern der Fall ist. Um dies zu erreichen, können Ressourcen besteuert und Arbeit entlastet, demokratische Unternehmensformen eingeführt, die Einkommens- und Vermögensungleichheiten verringert, Teilzeitarbeit gefördert, Werbung gemindert und Bildungsinhalte verändert werden. Darüber hinaus müssten der internationale Standortwettbewerb mitgedacht und internationale Handelsregeln angepasst werden.

\section{Anmerkungen}

[1] In der Literatur gibt es unterschiedliche Verständnisse einer PWÖ, dieser Artikel bezieht sich auf einen Teilbereich der Debatte.

[2] Die Klassifizierung ist hier stark vereinfacht und es gibt immer Vertreter(innen) aus den jeweiligen Schulen, die zu den hier geäußerten Verallgemeinerungen konträre Theorien entwickelt haben. Die Einteilung stimmt aber für die große Mehrheit der Theorien.

[3] Dies ist in Deutschland derzeit nur begrenzt der Fall. Grund ist die Verlagerung von Arbeiten, die geringe Qualifikation benötigen, ins Ausland.

[4] Daneben hätte die höhere Nachfrage nach Arbeit den Vorteil, dass Löhne steigen, die Lohnquote zunimmt und damit Einkommensungleichheiten tendenziell abnehmen würden.

\section{Literatur}

Acemoglu, D. (2002): Directed Technical Change. In: The Review of Economic Studies 69, 4/2002, S. 781-809.

Biesecker, A. (1999): Vorsorgendes Wirtschaften braucht Zeiten. Von einer "Ökonomie der Zeit“ zu „Ökonomien in Zeiten“. In: Spitzner, S. H. M. (Hrsg.): Zeitlandschaften: Perspektiven für eine öko-soziale Zeitpolitik. Stuttgart/Leipzig. S. 107-130.

Binswanger, H. (2013): The growth spiral: Money, energy, and imagination in the dynamics of the market process. Berlin, Heidelberg.

Braverman, H. (1998): Labor and monopoly capital: The degradation of work in the twentieth century. New York.

Burdín, G./Dean, A. (2012): Revisiting the objectives of worker-managed firms: An empirical assessment. In: Economic Systems 36, 1/2012. S. 158-171.

Galbraith, J./Crook, A. (1958): The affluent society. Boston 1958.
Jaffe, A./Newell, R./Stavins, R. (2003): Technological change and the environment. In: Handbook of Environmental Economics 1/2003. S. 461-516.

Krätke, M. (2009): Eine andere Demokratie für eine andere Wirtschaft: Wirtschaftsdemokratie und Kontrolle der Finanzmärkte. Widerspruch 28, 2/2009. S. 5-16.

Krugman, P. (1994): Competitiveness: a dangerous obsession. Foreign Affairs 73. S. 28.

Kümmel, R. (2011): The second law of economics: Energy, Entropy, and the Origins of Wealth. Berlin.

Ludewig, D./Meyer, B./Schlegelmilch, K. (2010): Nachhaltig aus der Krise: ökologische Finanzreform als Beitrag zur Gegenfinanzierung des Krisendefizits. In: Schriften zur Ökologie 9.

Marx, K. (1867): Das Kapital. Erster Band. MEW, Bd 23. Hamburg.

Paech, N. (2013): Suffizienz und Subsistenz: Therapievorschläge zur Überwindung der Wachstumsdiktatur. In: Konzeptwerk Neue Ökonomie (Hrsg.): Zeitwohlstand. München. S. 40-49.

Rajan, R. (2011): Fault lines: How hidden fractures still threaten the world economy. Princeton.

Rodrik, D. (2011): The globalization paradox: democracy and the future of the world economy. New York.

Schor, J. (1999): The Overspent American: Why. New York.

Solow, R. (1974): The economics of resources or the resources of economics. The American Economic Review 64, 2/1974. S. 1-14.

Stern, D. (1997): Limits to substitution and irreversibility in production and consumption: a neoclassical interpretation of ecological economics. Ecological Economics 21, 3/1997, S. 197-215.

Stiglitz, J. (2007): Making globalization work. New York.

Stockhammer, E. (2011): Peripheral Europe's debt and German wages: the role of wage policy in the Euro area. International Journal of Public Policy 7, 1/2011. S. 83-96.

\section{AUTOR + KONTAKT}

Steffen Lange, Dipl.-Volkswirt, ist Mitgründer des Konzeptwerks Neue Ökonomie und promoviert zum Thema "Macroeconomics of sustainable degrowth“.

Konzeptwerk Neue Ökonomie e. V., Klingenstr. 22, 04229 Leipzig. E-Mail: s.lange@knoe.org, Tel.: +4934139281686

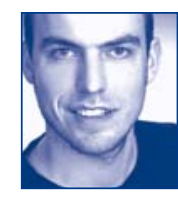

\section{Nachhaltigkeit}

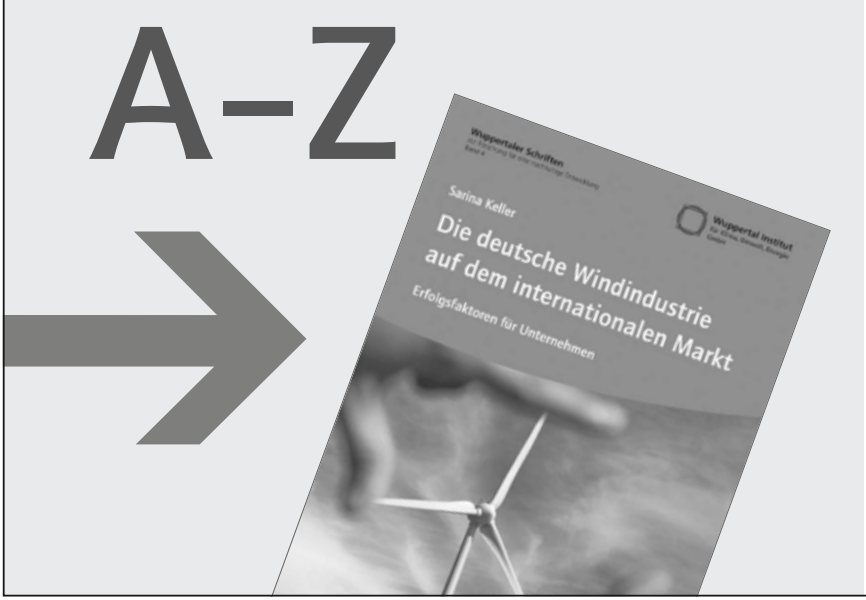

\section{E wie Erfolgsrezepte}

In den letzten zehn Jahren hat sich die weltweit installierte Windkraftleistung fast verzehnfacht. Gut fürs Klima - und lukrativ für Hersteller und Industrie. Während der bisherige Ausbau der Windenergie stark von deutschen und europäischen Unternehmen bestimmt wurde, sind inzwischen vor allem chinesische und amerikanische Unternehmen international führend. Zeit also, die Strategien und Erfolgsrezepte einer boomenden Branche zu prüfen.

\section{Sarina Keller}

Die deutsche Windindustrie auf dem internationalen Markt Erfolgsfaktoren fur Unternehmen

414 Seiten, broschiert, 39,95 Euro, ISBN 978-3-86581-659-7

\section{Erhältlich bei}

www.oekom.de | oekom@verlegerdienst.de

Die guten Seiten der Zukunft 